\title{
Series study of sub acute and chronic subdural haematoma
}

\author{
Abstract \\ Background: Subacute and chronic subdural haematoma is a common problem in the \\ elderly population. It occurs in the context of cerebral degeneration. Trauma, intracranial \\ hypotension, coagulopathy and chronic alcoholism are also predisposing factors. Clinical \\ profile may present similarly to stroke, raised intracranial pressure (ICP) or dementia. CT \\ and MRI are the diagnostic investigations. Burr hole craniostomy has good results in view \\ of improvement and recurrence. \\ Objective: The aim of this study is to determine the epidemiology, clinical profile, \\ optimum surgical procedure, postoperative course and recurrence of patients with SDH and \\ to compare with the literature.
}

Methods: This is a prospective series study of 50 cases. Multifactorial analysis was performed.

Results: Eighty four percent were older than 60 years. $10 \%$ were older than 90years. Trauma(42\%), coagulopathy (18\%) and alcoholism (12\%) were the risk factors found. $92 \%$ mimicked stroke in presentation. $56 \%$ had raised ICT. $14 \%$ were comatose. Burr hole drainage was done. It showed improvement in $92 \%$, recurrence in $16 \%$ and mortality in $6 \%$. Survival rate after 90 years was $60 \%$.

Conclusion: SDH is a problem common in elderly. Clinically it often mimics stroke. Burr hole craniostomy is optimum surgery for SDH. Surgery can be successful after 90 years also.

Keywords: Subdural haematoma, Subdural hygroma, Coagulopathy, Alcoholism, Burr hole craniostomy, Twist drill craniostomy
Volume 5 Issue 2 - 2016

\author{
Ajaya Kumar A, Grace Varghese, Litta \\ Thomas \\ Mar Gregorios Memorial Muthoot Medical Centre, India
}

Correspondence: Ajaya Kumar A, Chief consultant neurosurgeon, Mar Gregorios Memorial Muthoot Medical Centre, Kerala, Pin: 68964I, India, Tel 914682314000; 9447739838, Fax 9.14682E+I I, Email drjayakumara@gmail.com

Received: July 02, 2016 | Published: October 18, 2016

\section{Introduction}

Chronic subdural haematoma is defined as a fluid collection within the layers of duramater. ${ }^{1}$ It occurs predominantly in infancy and the elderly. ${ }^{2}$ SDH that develop from 3 days to 3 weeks after head injury are called subacute and those that appear later than 3 weeks after injury are called chronic. ${ }^{3}$

\section{Aim of study}

To study epidemiology, clinical profile, diagnosis, surgical treatment, improvement, recurrence and mortality of subacute and chronic subdural hematoma. Burr hole craniostomy is the surgical technique used and its efficacy is studied.

\section{Materials and methods}

Prospective study of 50 patients who underwent surgery for SDH is done. Factors analysed are age, sex, risk factors, comorbidities, clinical profile, CT findings, craniostomy, postoperative course and outcomes.

\section{Review of literature}

\section{Epidemiology}

Patients older than 40 years account for $80 \%$ of cases. ${ }^{1}$

Predisposing Factors: Cerebral atrophy, dehydration and degenerative cerebral diseases cause stretching of bridging veins. Low CSF pressure after a shunt or fistula also causes the same. ${ }^{4}$ Primary coagulopathy in children as well as secondary coagulopathy or anticoagulant treatment in adults are further risk factors. It has been shown that anticoagulant treatment in elder population increases the risk of developing chronic subdural haematoma. Chronic alcoholism causes propensity to experience trauma, hepatopathy, vascular fragility and brain atrophy. It is often cited as a major risk factor for $\mathrm{CSDH}^{4}$

\section{Pathophysiology}

All SDH probably start out as acute subdurals. Blood within the subdural space evokes an inflammatory response. Within days, fibroblasts invade the clot, and form neomembranes on inner (cortical) and outer (dural) surface. This is followed by in growth of neocapillaries, enzymatic fibrinolysis and liquefaction of blood clot. Fibrin degradation products are reincorporated into new clots and inhibit hemostasis. The course of CSDH is determined by the balance of plasma effusion and/or rebleeding from the neomembrane on the one hand and reabsorption of fluid on the other. ${ }^{3}$

\section{Symptoms and Signs}

Insidious onset is sometimes interpreted as dementia. In other patients, the onset of motor deficit is confused with cerebrovascular accident, transient ischemic attack or tumor. ${ }^{5}$ Common signs are hemiparesis, papilledema, hemianopsia, cranial nerve III abnormality and impaired consciousness. The clinical clue to the diagnosis of chronic subdural haematoma is the history of previous minor brain injury with other features such as the signs of hemispheric brain dysfunction (motor deficits, dysphasia etc.) and sometimes features of raised intracranial pressure (headache, vomiting and impaired level 
of conscuiousness).$^{6}$ In youngsters, seizure and altered behavior are often the presentation, especially in association with alcoholism. Common symptoms in the largest series of 2300 patients were refractory headache and sensorimotor and neuropsychiatric changes such as amnestic deficits or lack of concentration. ${ }^{7}$

\section{Diagnosis}

On CT and MRI, CSDH appears as a sickle shaped lesion between the cortical surface and calvarium. ${ }^{1}$ On CT scan, injuries 1-3 weeks old may be isodense with brain tissue. In this instance, I.V. contrast enhancement may delineate the cortical margin. Beyond 3 weeks subdural hematomas appear as a low density lesion. ${ }^{4}$ Bilateral isodense SDHs were likely to result in misdiagnosis in $\mathrm{CT}^{3}{ }^{3}$

CT classification according to Nomura and colleagues. ${ }^{1}$
Hyperdensity
Isodensity
Hypodensity
Mixed density
Layering type

Highest risk for recurrence is seen with a mixed density or layering type of haematoma on cranial CT because both types may be due to recent bleeding into cavity. MRI identifies and characterizes CSDH. MRI can demonstrate acute hemorrhage within CSDH and can differentiate CSDH from subdural hygroma. Recent literature questions the validity of assessing aging of mixed density subdural fluid collections on imaging. There is considerable CT evidence that some haematomas regress in size and do not need surgical treatment. ${ }^{5}$

\section{Medical Treatment}

Corticosteroid, long term mannitol and bedrest were included in conservative treatment. The effect of dexamethasone was thought to be anti inflammatory or antiangiogenic. ${ }^{1}$ Seizure prophylaxis should be started especially in alcohol abuse and age older than 65 years. If coagulopathy is present, it should be corrected, as it complicates neurosurgical treatment. ${ }^{6}$ Surgical evacuation of haematoma is indicated for symptomatic lesion (including focal deficit, mental status changes) and that with maximum thickness greater than or equal to $1 \mathrm{~cm}^{3}$

\section{Surgical options}

i. Placing two burr holes and irrigating through and through with tepid saline until the fluid runs clear. Classically CSDH contains dark motor oil fluid which does not clot.

ii. Single large burr hole with irrigation and aspiration $(5-30 \mathrm{~mm}$ diameter).

iii. Single burr hole drainage with placement of subdural drain.

iv. Twist drill craniostomy (up to diameter of $5 \mathrm{~mm}$ ) - has higher recurrence rate.

v. Formal craniotomy (larger than $30 \mathrm{~mm}$ ) with excision of subdural membrane, in cases with persistent recurrences after above procedures probably due to seepage from subdural membrane and for organized, solid SDH.

Techniques that promote continued drainage and that may reduce residual fluid and prevent reaccumulation are using a generous burr hole under the temporal is muscle and use of subdural drain.
Twist drill craniostomy may be performed at beside under local anaesthesia. ${ }^{8}$ It is useful in patients with multiple comorbidities who cannot undergo a surgical treatment. A ventricular catheter is inserted into the subdural space and is drained into a standard ventriculostomy drainage bag maintained $20 \mathrm{~cm}$ below the level of craniostomy site. Irrigation reduces recurrence.

Burr holes are recommended to be generous $(>2.5 \mathrm{~cm})$. A subtemporal craniectomy should be performed, and bipolar coagulation is used to shrink the edges of dura and subdural membrane back to the full width of bony opening. This allows continued drainage of fluid into the temporalis muscle. A piece of gel foam may be placed over the opening to help prevent fresh blood from oozing into the opening. ${ }^{3}$

Subdural drain is associated with a decrease in need for repeat surgery. ${ }^{8}$ A closed drainage system is recommended. Burr hole craniostomy is the most commonly used technique. Burr hole shares the advantages of twist drill, a high cure rate and safety and the advantage of craniotomy, high efficiency. ${ }^{1}$ Twist drill and burr hole craniostomy can be considered first tier treatment, while craniotomy may be used as second tier treatment. ${ }^{8}$

Another surgical technique described is small temporal craniotomy with the dura left open and the subdural space communicating with the subtemporalis area. ${ }^{7}$

\section{Outcome}

Residual subdural fluid collections are common. CTs showed persistent fluid in $78 \%$ of cases on postoperative day 10 and $15 \%$ after 40 days, and may take up to 6 months for complete resolution. ${ }^{3}$

\section{Complications of surgical treatment}

Seizure can occur if inner membrane is torn. If decompression is rapid, hyperemia can occur and cause intracerebralhemorrhage. ${ }^{5}$ Failure of brain to re expand may lead to recurrence. Generous hydration of patients with intravenous fluids postoperatively was thought to increase brain volume and decrease risk of recurrence. ${ }^{1}$ Some amount of pneumocephalus is common in immediate postoperative CT scan. ${ }^{9}$ Tension pneumocephalus can cause worsening and may require aspiration. Subdural empyema has been reported after SDH evacuation.

The mortality is less than 10 percent. About 80 percent are able to resume normal functioning. ${ }^{5}$ Mc Kissock reported 13\% mortality in patients who are comatose at the time of operation and 5\% in those who are alert.

\section{Analysis and results}

Youngest patient is of age 14yr, who had underwent VP shunt for aqueductal stenosis Oldest patient is of age 98, after a fall. Most patients are after 60 years- 42 viz. $84 \%$. Five (10\%) patients are after 90 years. Males are $35(70 \%)$, and females 15(30\%).

Risk factors assessed are coagulopathy, trauma, alcoholism and Ventriculoperitoneal shunt. Coagulopathy is secondary to drugs such as antiplatelets. Twenty one $(42 \%)$ had history of obvious trauma. Nine (18\%) were consuming antiplatelet drugs. Six (12\%) were alcoholics. One was following VP shunt. Comorbidities were hypertension in $29(58 \%)$, diabetes mellitus in $21(42 \%)$, respiratory disease in $10(20 \%)$ and cardiac disease in $7(14 \%)$.

Clinical manifestations were like cerebrovascular accident in $46(92 \%)$ with weakness or aphasia. Twenty eight $(56 \%)$ had features of raised intracranial tension such as headache, vomiting, 
papilledema. Consciousness level was altered in 13(26\%). Three (6\%) had dementia. Glasgow Coma Scale was 15 in 37(74\%). Fourty three (86\%) had GCS of 9 and above. Seven (14\%) had GCS of 8 and below.

CT brain is the main investigation for diagnosis. MRI is done if there is doubt because of isodensity or bilaterality. Subacute SDH is isodense with grey matter in CT, chronic SDH is hypodense and acute on chronic SDH is mixed. Twenty eight (56\%) had isodense SDH, $14(28 \%)$ had mixed density SDH and $8(16 \%)$ had hypodense SDH (Figure 1). Twenty six (52\%) patients had midline shift of greater than $1 \mathrm{~cm}$. Twenty one (42\%) had left sided SDH, 16(32\%) had right sided SDH and 13 (26\%) had bilateral haematoma (Figure 2). Necessity for two burr holes was decided by the size and extent of SDH. For haematoma extending over entire convexity, two burr holes are made. Posterior burr hole is enlarged into craniectomy of size $2.5-3 \mathrm{~cm}$. For making craniectomy of size $3 \mathrm{~cm}$, two small holes can be made with perforator and connected with craniotome. Burr holes were made in anterior and posterior regions in $31(62 \%)$ patients. Fluid pressure on opening was very high (jet like) in 20(40\%). Membranes were present in $16(32 \%)$. There was active bleeding in $8(16 \%)$ patients. It was controlled by iso cool cautery and gelfoam. Lavage with antibiotic saline was done till effluent was clear. Endoscopic examination was done to assess membranes. Gelfoam was placed in hole to prevent blood oozing in from wound. Nonsuction drain was inserted in all cases. Amount of drain was greater than $100 \mathrm{ml}$ in $7(14 \%)$ (Figure $4-8)$.

Postoperative CT brain was taken routinely. One (2\%) had acute bleeding and worsening. He was consuming antiplatelet drug. Decompressive Craniectomy was done and he survived with ventilation and supportive care. One (2\%) had tension pneumocephalus, which was aspirated. Mortality was $3(6 \%)$. All of them were due to respiratory complications. There were $5(10 \%)$ above age of 90 years and 3 survived (Figure 9-11). Fourty six (92\%) had improvement in neurological status. One (2\%) remained in same status. Eight (16\%) patients had symptomatic recurrence and were managed by aspiration under local anaesthesia.

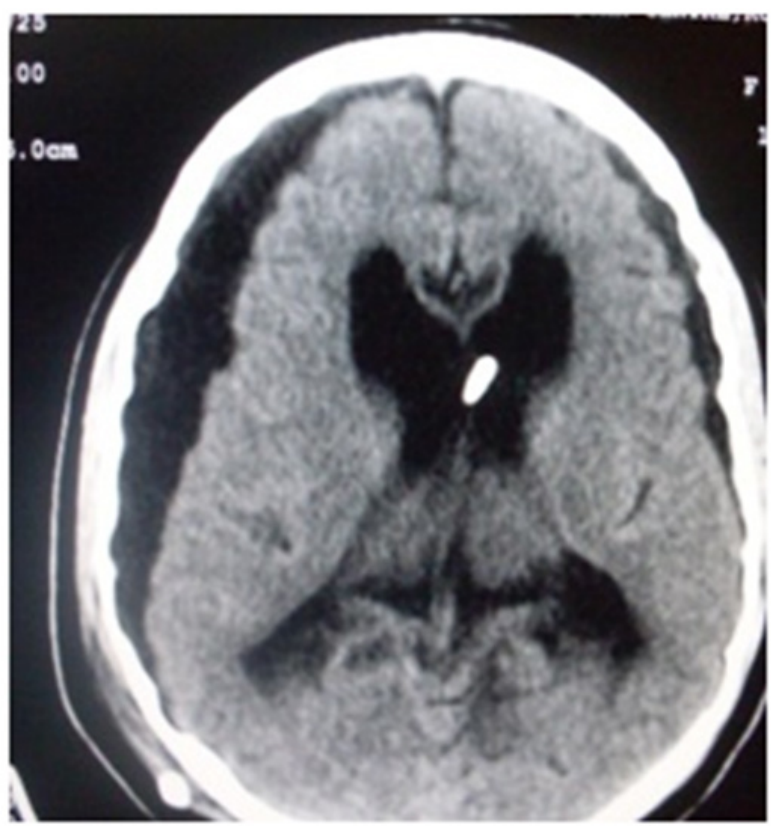

Figure I CT brain of $14 \mathrm{yr}$ girl with hypodense SDH after VP shunt.

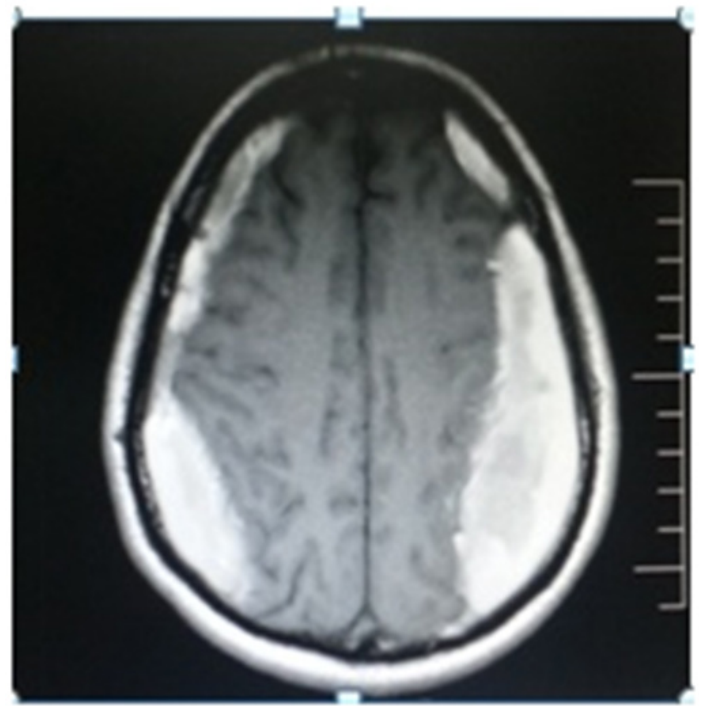

Figure 2 MRI brain showing bilateral SDH.

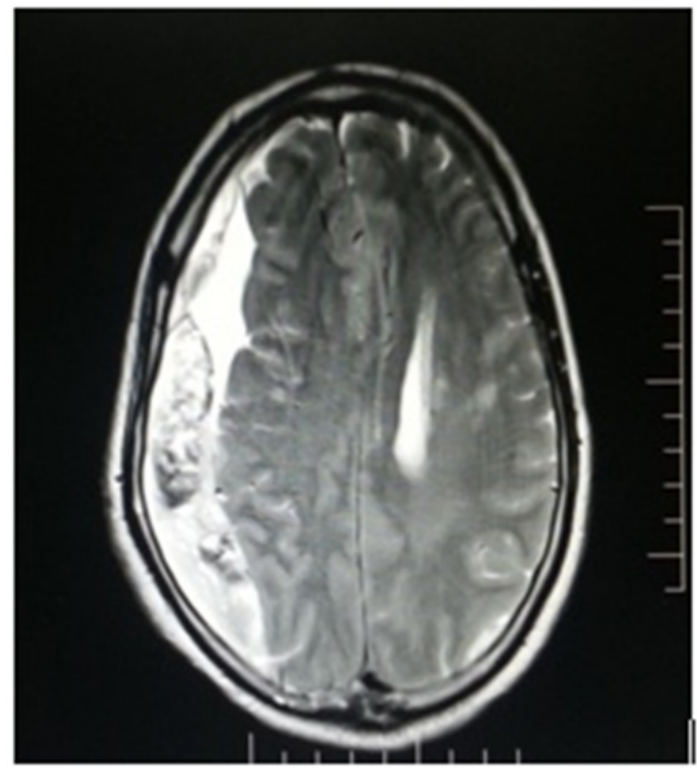

Figure 3 MRI brain showing layering.

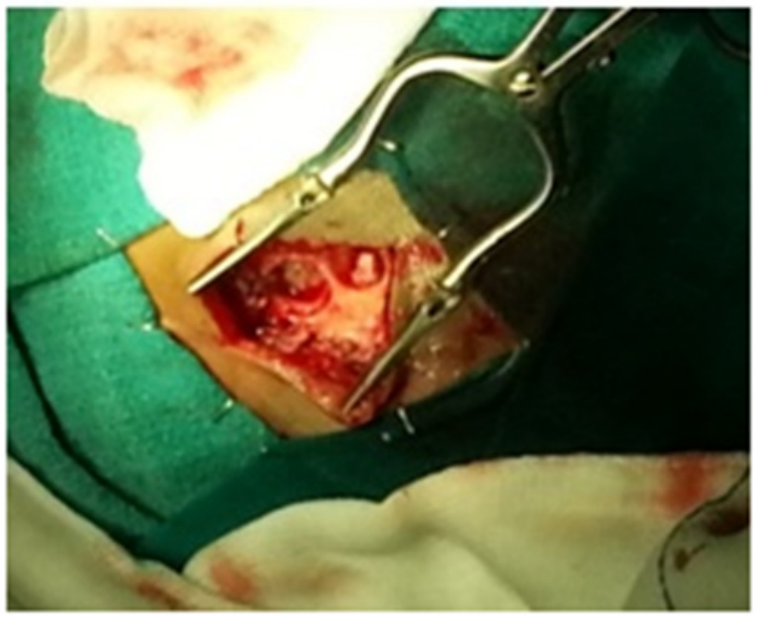

Figure 4 Two small burr holes are made with perforator. 


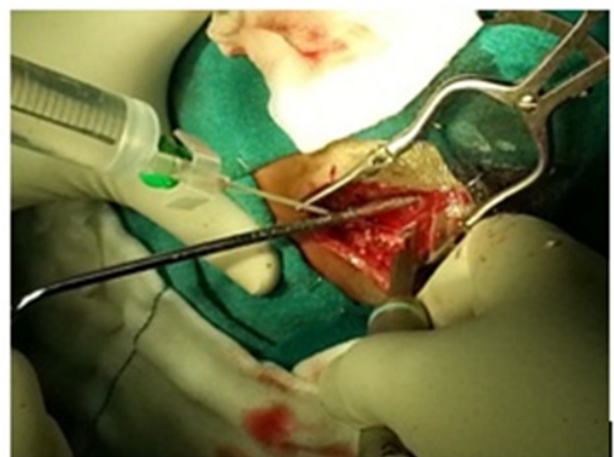

Figure 5 Small holes are connected with craniotome to make craniostomy.

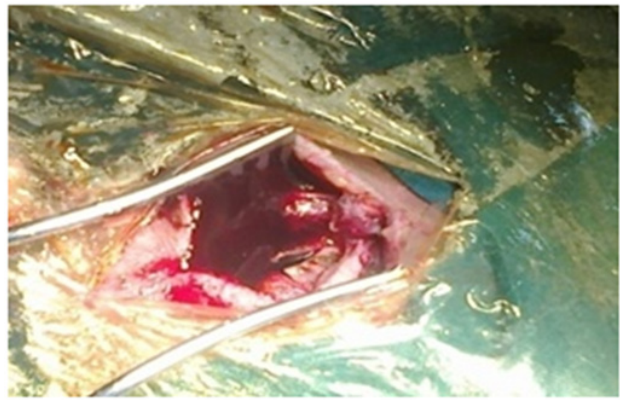

Figure 6 Dark altered blood coming from dural opening.

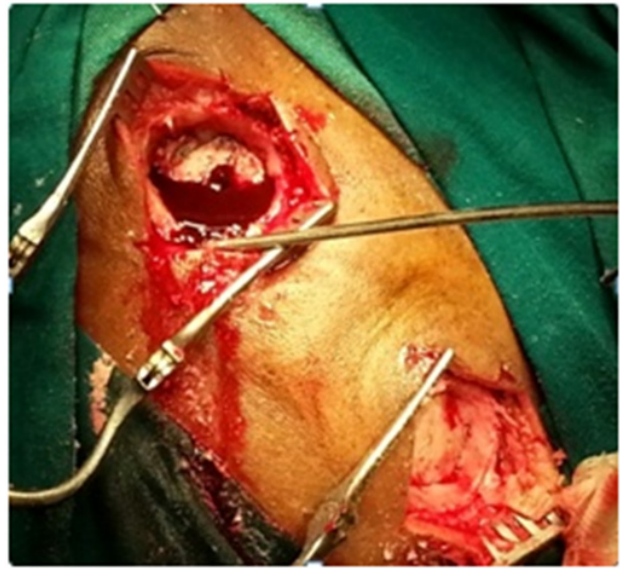

Figure 7 Two burr hole craniostomy drainage.

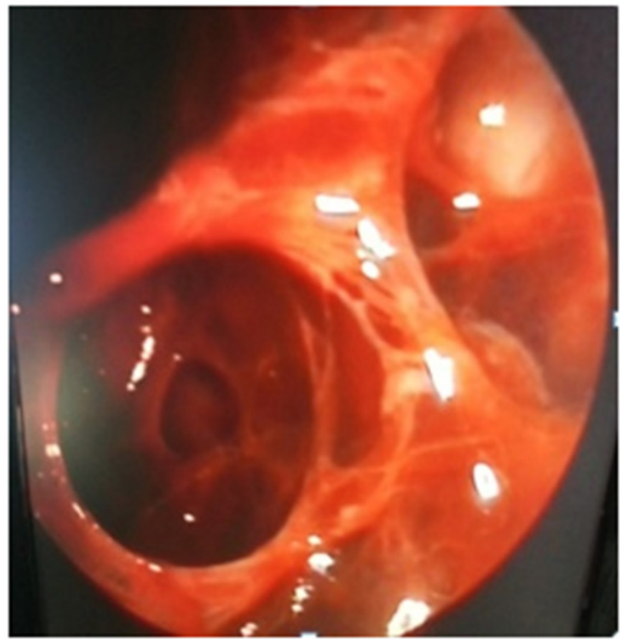

Figure 8 Endoscopic view of membranes.

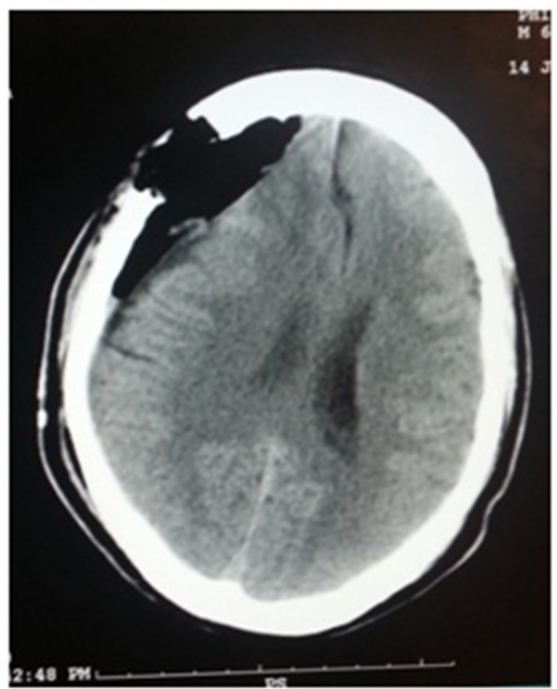

Figure 9 Postoperative CT showing near total evacuation of SDH and presence of pneumocephalus.

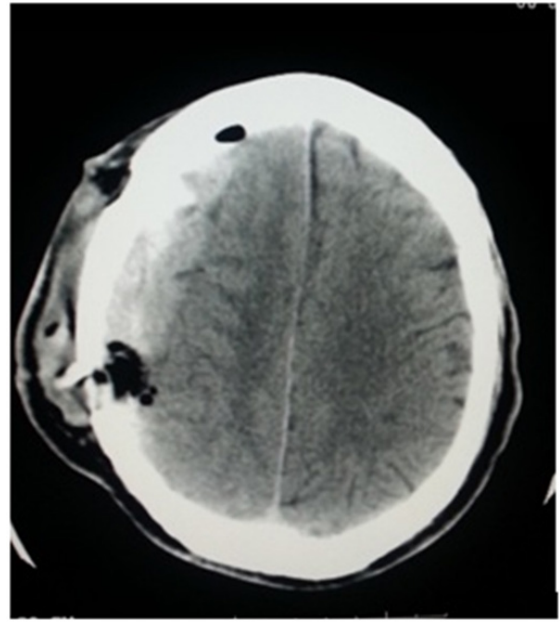

Figure 10 Postoperative CT showing acute bleed in patient who was consuming antiplatelet.

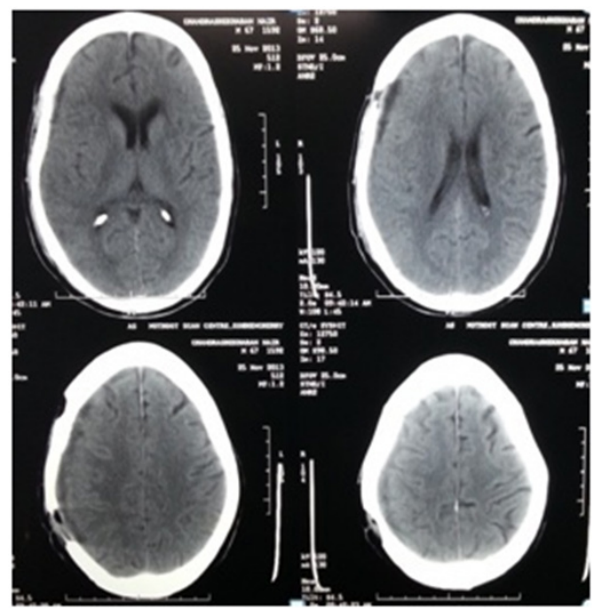

Figure I I Postoperative CT showing complete resolution.

Glasgow Outcome Scale is as follows-

1 (died) $-3(6 \%)$

Citation: Ajaya KA, Grace V, Litta T. Series study of sub acute and chronic subdural haematoma.J Neurol Stroke. 20I6;5(2): I-6.

DOI: I0.I5406/jnsk.2016.05.00I68 
4 (moderate disability but independent) - 14(28\%)

5 (good recovery) - 33(66\%).

\section{Discussion}

Subacute and Chronic SDH is more common in elderly. Eighty four percent were after 60 years of age. Seventy percent patients are males. Surgery can be done even after 90 years. Risk factors for developing CSDH are coagulopathy, trauma, alcoholism and intracranial hypotensive state such as after VP shunt. It is common in elderly consuming antiplatelet drugs and alcoholics after a trivial trauma. Although only $42 \%$ gave history of trauma, in other patients the trauma may have been too trivial to be noticed. Although common comorbidities were diabetes mellitus and hypertension, respiratory problems such as COPD, aspiration pneumonia had impact on recovery. Ninety two percent had presentation like stroke. Features of raised ICP were present in $56 \%$. Deterioration of consciousness was present in $26 \%$. Dementia was present in $6 \%$ and it was in bilateral disease.

CT was the primary investigating modality. Isodensity suggestive of subacute $\mathrm{SDH}$ was present in $56 \%$ and mixed density suggestive of acute on chronic SDH was present in $28 \%$. Hypodense collection of CSDH was seen only in $16 \%$. Although the surgical options vary from twist drill craniostomy to craniotomy, burr hole craniostomy was the technique used. For large hematomas, anterior and posterior holes were made. For making the optimum craniostomy of size $3 \mathrm{~cm}$, two small holes were made with perforator and connected with craniotome.

Ninety two percent had neurological improvement $(80 \%$ in literature). Mortality was only $6 \%$ ( $10 \%$ in papers). Recurrence rate was $16 \%$. Survival rate above 90 years was $60 \%$. This study emphasizes the efficacy of burr hole craniostomy for subacute and chronic subdural haematoma and success of surgery after the age of 90years.

\section{Conclusion}

Subacute and chronic subdural haematoma is a disease of elderly. It is common in those with coagulopathy and cerebral degeneration. Clinical profile usually mimics stroke or dementia. MRI will demonstrate clearly in case of doubt in CT. Burr hole craniostomy is the preferred surgery. Result of properly planned surgery is good. Associated systemic diseases have to be controlled. Independent good recovery is possible in most cases. Surgery can be done successfully even after 90 years of age.

\section{Acknowledgments}

None.

\section{Conflicts of interest}

None.

\section{References}

1. Joachim K Krauss, Lawrence F Marshall, Ralf Weigel. Medical and surgical management of chronic subdural hematomas.Youmans Neurological surgery, (6th edn), Chapter 38. 2011;pp.535-543.

2. Adhiyaman V, Asghar M, Ganeshram KN, et al. Chronic subdural haematoma in the elderly. Postgrad Med J. 2002;78:71-75.

3. Mark S Greenberg Chronic subdural hematoma: hand book of neurosurgery. (7th edn). 2002;p.899-902.

4. Kenneth W. Lindsay, Ian Bone, et al. Chronic subdural hematoma: Neurology and Neurosurgery illustrated. 2010;pp.239-240.

5. Srinath Samudrala, Raul R Cooper, Robert $H$ Wilkins, et al Traumatic intracranial hematomas: Neurosurgery. (2nd edn), Vol 2. 1996;pp.2797-2807.

6. Iliescu IA. Current diagnosis and treatment of chronic subdural haematomas. J Med Life. 2015;8(3): pp.278-284

7. Sambasivan M. An overview of chronic subdural haematoma, experience with 2300 cases. Surg Neurol. 1997;47:418-422.

8. Weigel R, Schmiedek P, Krauss J. Outcome of contemporary surgery for chronic subdural haematoma- evidence based review. $J$ Neurol Neurosurg Psychiatry. 2003;74(7):937-943.

9. Ihab Z. Pneumocephalus after surgical evacuation of chronic subdural hematoma- Is it a serious complication?. Asian $J$ Neurosurg. 2012;7(2):66-74. 